Palavras chave:

llex paraguariensis

Ácido Indol Butírico

Enraizamento

Maturação

Revigoramento

Histórico:

Recebido 22/07/2014

Aceito 14/09/2015

Keywords:

llex paraguariensis

Indolbutiric Acid

Rooting

Maturation

Reinvigoration

Correspondência: carlos.stuepp@ufpr.br
Carlos André Stuepp', Juliany de Bitencourt', Ivar Wendling², Henrique Soares Koehler', Katia Christina Zuffellato-Ribas'

\title{
PROPAGAÇÃO DE ERVA-MATE UTILIZANDO BROTAÇÕES DEANELAMENTO E DECEPA EM MATRIZES DE DUAS IDADES
}

RESUMO: A erva-mate é conhecida por seu uso na forma de infusões, como chás e chimarrão. Porém, há um vasto campo de aplicação desta espécie, principalmente na indústria de cosméticos e medicamentos. Um dos fatores limitantes à clonagem comercial de erva-mate é a falta de métodos eficientes de rejuvenescimento de material adulto. Propágulos juvenis podem ser obtidos por meio de anelamento ou decepa de árvores adultas. Desta forma, o objetivo deste trabalho foi verificar a capacidade de enraizamento de estacas oriundas de brotações de 12 meses de idade provenientes de anelamento e decepa de árvores de erva-mate com 17 anos e mais de 80 anos, realizados no inverno/2006 e verão/2007 e tratadas com ácido indol butírico (AIB), nas concentrações de 0 e 3000 $\mathrm{mg} \cdot \mathrm{L}^{-1}$. O plantio foi realizado em caixas plásticas preenchidas com vermiculita e casca de arroz carbonizada na proporção de I:I ( $/ / \mathrm{v})$ e após 90 dias em casa de vegetação, foram avaliadas as seguintes variáveis: porcentagem de estacas enraizadas, número de raízes/ estaca, comprimento das três maiores raízes/estaca, porcentagem de estacas vivas, com calos e mortas. $O$ enraizamento não foi influenciado pela origem da brotação (anelamento ou decepa). Porém, as brotações obtidas das matrizes mais jovens, assim como aquelas coletadas no verão, apresentaram melhores resultados. Concluiu-se que a estaquia realizada com brotações rejuvenescidas por anelamento ou decepa é uma técnica viável para a otimização do enraizamento de estacas de erva-mate.

\section{PROPAGATION OF YERBA MATE USING SHOOTS FROM GIRDLING AND COPPICING FOR TWO STOCK PLANTS AGES}

ABSTRACT: "Yerba mate" is well know for its use as an infusion, either as tea or "chimarrão". However, there are many other potential applications for this species, mainly in the cosmetic and drug industries. One limiting factor to the commercial vegetative propagation of "Yerba mate" is the lack of efficient methods of rejuvenation for mature plants. Juvenile propagules can be obtained by stem girdling or coppicing of mature trees. The main objective of this work was to verify the rooting ability of cuttings from 12 months old sprouts, generated after girdling and coppicing of 17 years and 80 years old trees, during winter/2006 and summer/2007 and treated with indolbutiric acid (IBA) with two concentrations of 0 and $3000 \mathrm{mg} \cdot \mathrm{L}^{-1}$. Planting was made in plastic containers filled with vermiculite and carbonized rice bark, in a I:I ratio and after 90 days in the greenhouse, the following variables were evaluated: percentage of cuttings with roots, average number of roots per cutting, length of the three longest roots per cutting and percentage of alive cuttings, with callus and dead. Results showed that rooting was not influenced by the origin of sprouts (girdling or coppicing). However, branches obtained by more juvenile trees, as well as those collected during summer, presented better results. Thus was concluded that stem cuttings with branches from girdling or coppicing is a viable technique to optimize rooting of "Yerba mate" cuttings.

' Universidade Federal do Paraná - Curitiba, Paraná, Brasil

2 Empresa Brasileira de Pesquisa Agropecuária, Centro Nacional de Pesquisa de Florestas - Colombo, Paraná, Brasil 


\section{INTRODUÇÃO}

A erva-mate (llex paraguariensis), utilizada principalmente na forma de infusões quentes ou frias, como o chimarrão, tereré e chá-mate, apresenta uma vasta aplicação comercial, utilizada para a produção de corantes, sorvetes, caramelos, em medicamentos para tratamento de hipertensão, bronquite e pneumonia, produtos de uso pessoal, como perfumes e desodorantes, dentre outros (PAGLIOSA et al., 20I0; DARTORA et al., 20l3).

Com relação à propagação vegetativa via estaquia, a erva-mate é uma espécie de difícil enraizamento, de modo que os fatores que afetam este processo devem ser considerados e otimizados para o sucesso da técnica. Entre estes fatores está a idade ontogenética da planta matriz (WENDLING et al., 20l4a), que pode influenciar negativamente o enraizamento, sendo necessário o rejuvenescimento prévio para que se obtenham resultados satisfatórios (WENDLING et al., 2007; WENDLING et al., 20l4b).

Uma das principais limitações à produção de mudas de erva-mate em escala comercial está relacionada à carência de métodos eficientes de rejuvenescimento de material adulto (WENDLING et al., 2007; BRONDANI et al., 2008). Assim, o entendimento do processo de maturação das plantas pode incrementar as perspectivas de sucesso na clonagem de árvores adultas, trazendo maior eficiência para a seleção, melhoramento e, sobretudo, beneficiando a silvicultura clonal da espécie (WENDLING et al., 20l4b).

Diversos trabalhos vêm conotando a importância da juvenilidade na propagação de ervamate (BRONDANI et al., 2008; BITENCOURT et al., 2009; WENDLING et al., 2013). Em experimento com miniestaquia de erva-mate, Brondani et al. (2008) concluíram que os propágulos de origem juvenil foram superiores na porcentagem de enraizamento, número e comprimento de raízes, quando comparados com propágulos adultos da mesma espécie.

Uma das formas de resgate de plantas adultas comumente utilizada é a decepa para indução de brotações epicórmicas (STUEPP etal., 20 I4). Estas brotações possuem características morfológicas e fisiológicas de plantas juvenis, de fundamental importância à recuperação da capacidade de enraizamento (MELO et al., 20I2; WENDLING et al., 20I3). De acordo com Almeida et al. (2007), a decepa de plantas adultas de Eucalyptus cloeziana se apresentou mais eficiente do que o anelamento na emissão de brotações e enraizamento de estacas.

Assim, o presente trabalho teve como objetivo verificar a influência da origem da brotação, idade da planta matriz, uso da auxina (AIB) e época de coleta no enraizamento de estacas oriundas de brotações provenientes de anelamento e decepa de erva-mate.

\section{MATERIAL E MÉTODOS}

\section{Caracterização geral e tratamentos}

O experimento foi conduzido em casa de vegetação do Laboratório de Propagação de Espécies Florestais da Embrapa Florestas (Colombo-PR) entre julho de 2007 (inverno) e março de 2008 (outono). As estacas foram confeccionadas a partir de ramos provenientes de rebrota de anelamento e decepa, realizadas em árvores de 17 anos (Colombo-PR) e árvores com mais de 80 anos (São Mateus do Sul-PR).

$O$ anelamento foi realizado por meio da remoção de um anel de casca com cerca de $1,5 \mathrm{~cm}$ de largura, sem danificar o lenho; a decepa constituiu na derrubada das árvores a $15 \mathrm{~cm}$ do solo. Ambos os processos foram realizados no inverno de 2006 e uma duplicata foi realizada, apenas em árvores com mais de 80 anos no verão/2007. Após 12 meses foram coletadas brotações para a realização dos experimentos.

As estacas foram confeccionadas com cerca de $12 \mathrm{~cm}$ de comprimento e duas folhas com sua área reduzida à $50 \%$ da superfície original, corte em bisel na base e reto no ápice. A desinfestação foi realizada com hipoclorito de sódio à $0,5 \%$ por 5 minutos, seguida de lavagem em água corrente por 5 minutos. As bases das estacas foram imersas em solução com fungicida sistêmico (Benomyl ${ }^{\circledR}$ - I,0 g. $\left.\mathrm{L}^{-1}\right)$ por 15 minutos e, posteriormente, tratadas com AIB (ácido indol butírico) em solução hidroalcoólica $(50 \% \mathrm{v} / \mathrm{v})$ por 10 segundos, nas concentrações de 0 e $3000 \mathrm{mg} \cdot \mathrm{L}^{-1}$. O plantio foi realizado em caixas plásticas, utilizando casca de arroz carbonizada e vermiculita de granulometria média como substrato, na proporção de I:I (v/v).

Após 90 dias em casa de vegetação climatizada com nebulização intermitente (temperatura de $24{ }^{\circ} \mathrm{C}$ $\pm 2{ }^{\circ} \mathrm{C}$ e umidade relativa do ar superior a $80 \%$ ), foram avaliadas as seguintes variáveis: porcentagem de estacas enraizadas (consideradas aquelas com presença de raízes a partir de $1 \mathrm{~mm}$ de comprimento); número de raízes por estaca; comprimento das três maiores raízes por estaca; porcentagem de estacas com calos (consideradas as estacas vivas, com formação de calos e sem formação de raízes); porcentagem de estacas vivas (consideradas as estacas vivas, sem formação de 
calos e sem formação de raízes) e porcentagem de estacas mortas.

\section{Análise estatística}

Brotações provenientes de anelamento e decepa coletadas no inverno/2006

Foram comparados os dados obtidos da estaquia de ramos com 12 meses, provenientes de anelamento e decepa em árvores de 17 anos e árvores de mais de 80 anos. Utilizaram-se duas concentrações do regulador vegetal: 0 e $3000 \mathrm{mg} \cdot \mathrm{L}^{-1}$ de AIB.

Os dados foram analisados segundo um delineamento inteiramente casualizado, com arranjo fatorial $(2 \times 2 \times 2)$ com dois métodos de indução de brotações, duas idades de plantas matrizes e duas concentrações de AIB, com 4 repetições de 20 estacas por unidade experimental.

\section{Brotações provenientes de decepa realizada em árvores de mais de 80 anos}

Foram comparados os dados obtidos da estaquia de ramos de 12 meses, provenientes de decepa realizada em árvores de mais de 80 anos no inverno/2006 e verão/2007. Os tratamentos consistiram da aplicação de 0 e $3000 \mathrm{mg} \cdot \mathrm{L}^{-1}$ de AIB.

Os dados foram analisados segundo um delineamento inteiramente casualizado, com arranjo fatorial $(2 \times 2)$, testando-se duas épocas da instalação da decepa e duas concentrações de AIB, com 4 repetições de 20 estacas por unidade experimental.

\section{Brotações provenientes de anelamento e decepa coletadas no verão/2007}

Foram comparados os dados obtidos da estaquia de ramos de 12 meses, provenientes de anelamento e decepa realizados no verão/2007, em árvores de mais de 80 anos de idade.

As estacas provenientes de decepa foram suficientes para a realização de dois tratamentos com AIB: 0 e $3000 \mathrm{mg} \cdot \mathrm{L}^{-1}$. As estacas provenientes de anelamento não foram suficientes, de modo que foi realizado o plantio das estacas sem aplicação do regulador vegetal. Assim, os tratamentos ( $T$ ) foram considerados da seguinte maneira: TI: estacas provenientes de anelamento; T2: estacas provenientes de decepa, com aplicação de $0 \mathrm{mg} \cdot \mathrm{L}^{-}$ I de AIB; T3: estacas provenientes de decepa, com aplicação de $3000 \mathrm{mg} \cdot \mathrm{L}^{-1}$ AIB.

Os dados foram analisados segundo um delineamento inteiramente casualizado, com 3 tratamentos e 4 repetições de 18 estacas por unidade experimental.

Os dados coletados foram submetidos à análise de variância. Inicialmente, as variâncias dos tratamentos foram analisadas quanto a sua homogeneidade pelo teste de Bartlett. As variáveis cujas variâncias mostraram-se homogêneas tiveram as médias dos tratamentos testadas por meio do teste de F. Quando os resultados revelaram existir diferenças significativas entre as médias dos tratamentos, estas foram comparadas pelo teste de Tukey a 5\% de probabilidade (BITENCOURT et al., 20I0).

\section{RESULTADOS E DISCUSSÃO}

\section{Brotações provenientes de anelamento e decepa coletadas no inverno/2006}

A análise de variância (ANOVA) revelou interação entre os fatores idade da planta matriz e aplicação de regulador vegetal para a porcentagem de enraizamento, comprimento das três maiores raízes e sobrevivência. Houve interação entre os fatores origem dos ramos e aplicação de regulador vegetal para o número de raízes e sobrevivência, e interação entre os fatores origem dos ramos e idade da planta matriz para a porcentagem de mortalidade.

No tratamento testemunha, as estacas provenientes de matrizes com 17 anos apresentaram maior porcentagem de enraizamento do que aquelas provenientes de matrizes com mais de 80 anos. Para as matrizes de 17 anos, o tratamento com regulador vegetal não diferiu estatisticamente da testemunha, enquanto para as matrizes de 80 anos, o tratamento com $3000 \mathrm{mg} \cdot \mathrm{L}^{-1}$ de AIB foi superior à testemunha (Tabela I).

Não foi observada influência da origem das brotações no enraizamento das estacas, porém, em experimento realizado com Eucalyptus cloeziana, Almeida et al. (2007) observaram que estacas provenientes de brotações de anelamento não responderam ao enraizamento, enquanto as brotações de cepa foram mais eficientes.

material proveniente de árvores de 17 anos apresentou maiores porcentagens de enraizamento sem a utilização de AIB, ao passo que o material oriundo de matrizes com mais de 80 anos enraizou melhor com a utilização do regulador vegetal (Tabela I). 
TABELA I Porcentagem de enraizamento em estacas de erva-mate provenientes de anelamento e decepa, em duas idades de plantas matrizes e dois tratamentos com regulador vegetal, realizados no inverno/2006, Colombo-PR.

TABLE I Percentage of rooting in cuttings of yerba mate from girdling and coppicing in two ages of mother plants and two plant growth regulator treatments, conducted in winter/2006, Colombo-PR.

\begin{tabular}{ccc}
\hline \multirow{2}{*}{ Idade matriz } & \multicolumn{2}{c}{$\%$ Enraizamento } \\
\cline { 2 - 3 } & $0 \mathrm{mg} \cdot \mathrm{L}^{-1}$ AIB & $3000 \mathrm{mg} \cdot \mathrm{L}^{-1}$ AIB \\
\hline I7 anos & $71,25 \mathrm{a} \mathrm{A}$ & $61,88 \mathrm{a} \mathrm{A}$ \\
80 anos & $50,63 \mathrm{~b}$ B & $70,63 \mathrm{a} \mathrm{A}$ \\
\hline
\end{tabular}

Coeficiente de variação $(\%)=22,40$

Médias seguidas da mesma letra minúscula na vertical e médias seguidas da mesma letra maiúscula na horizontal, não diferem significativamente entre si pelo teste de Tukey a $5 \%$ de probabilidade.

Estacas coletadas de matrizes com 17 anos apresentaram maior número de raízes por estaca diferindo estatisticamente das matrizes com mais de 80 anos de idade (Tabela 2). Da mesma forma, Bitencourt et al. (2009), verificaram que o material proveniente de brotações rejuvenescidas apresentou os melhores resultados quanto a porcentagem de estacas enraizadas, assim como de número e comprimento de raízes. $O$ uso de brotações proveniente de revigoramento de plantas adultas tende a propiciar uma maior velocidade e qualidade de enraizamento, refletindo na qualidade da muda produzida (PIJUT et al., 20I I; WENDLING et al., 20I4b).

TABELA 2 Número de raízes por estaca de erva-mate provenientes de anelamento e decepa, em duas idades de plantas matrizes e dois tratamentos com regulador vegetal, realizados no inverno/2006, Colombo-PR.

TABLE 2 Number of roots per cutting of yerba mate from girdling and coppicing in two ages of mother plants and two plant growth regulator treatments, conducted in winter/2006, Colombo-PR.

\begin{tabular}{|c|c|c|c|c|}
\hline \multicolumn{5}{|c|}{ Número de raízes (n) } \\
\hline Idade matriz & $\operatorname{AlB}\left(m g \cdot L^{-1}\right)$ & Anelamento & Decepa & Médias \\
\hline 17 anos & 0 & 8,95 & 13,27 & $\mathrm{II}, 70 \mathrm{a}$ \\
\hline 17 anos & 3000 & 11,83 & 12,74 & \\
\hline 80 anos & 0 & 7,18 & 10,18 & $9,75 \mathrm{~b}$ \\
\hline 80 anos & 3000 & 11,03 & 10,60 & \\
\hline \multicolumn{2}{|c|}{ Concentrações AIB $\left(\mathrm{mg} \cdot \mathrm{L}^{-1}\right)$} & Anelamento & Decepa & Médias \\
\hline \multicolumn{2}{|c|}{0} & 8,07 b B & 11,73 a A & 9,90 \\
\hline \multicolumn{2}{|c|}{3000} & $\mathrm{I} I, 43$ a $\mathrm{A}$ & I I,67 a A & 11,55 \\
\hline
\end{tabular}

Médias seguidas da mesma letra minúscula na vertical e médias seguidas da mesma letra maiúscula na horizontal, não diferem significativamente entre si pelo teste de Tukey a $5 \%$ de probabilidade.
Para o número de raízes por estaca, pode-se verificar na concentração de $0 \mathrm{mg} \cdot \mathrm{L}^{-1}$ de AlB que as estacas provenientes de decepa apresentaram resultado superior àquelas provenientes de anelamento. Para as estacas provenientes de anelamento, a concentração de 3000 $\mathrm{mg} \cdot \mathrm{L}^{-1}$ de $A \mathrm{AlB}$ foi superior à testemunha. $\mathrm{O}$ maior valor encontrado para esta variável foi o de 13,27 raízes, em estacas provenientes de decepa de plantas de 17 anos, sem aplicação de regulador vegetal (Tabela 2).

O comprimento das raízes por estaca foi maior para as matrizes com 17 anos, diferindo significativamente daquelas com 80 anos (Tabela 3). 0 maior vigor atribuído às plantas mais jovens tem sido considerado um fator preponderante no sucesso da propagação vegetativa (TITON et al., 2006; WENDLING et al., 20l4b). Pode estar associado a presença de inibidores e diminuição no conteúdo de co-fatores, à medida que a planta vai se tornando adulta, refletindo também, na qualidade do sistema radicial (HUSEN, 20I2).

O tratamento com AlB favoreceu o aumento no comprimento das raízes, porém não diferiu estatisticamente da testemunha. Sabe-se que a auxina é importante para o estímulo da indução radicial (POP et al., 20I I), de modo que as estacas tratadas com AIB podem ter iniciado o processo de enraizamento antes daquelas não tratadas, justificando a tendência ao maior comprimento das raízes observado.

TABELA 3 Comprimento médio das três maiores raízes por estaca de erva-mate, provenientes de anelamento e decepa, em duas idades de plantas matrizes e dois tratamentos com regulador vegetal, realizados no inverno/2006, Colombo-PR.

TABLE 3 Average length of the three longest roots per cutting of yerba mate from girdling and coppicing in two ages of mother plants and two plant growth regulator treatments, conducted in winter/2006, Colombo-PR.conducted in winter/2006, Colombo-PR.

Comprimento médio das três maiores raízes por estaca $(\mathrm{mm})$

\begin{tabular}{|c|c|c|c|c|c|}
\hline \multirow{2}{*}{$\begin{array}{l}\text { Idade } \\
\text { matriz }\end{array}$} & \multicolumn{2}{|c|}{$0 \mathrm{mg} \cdot \mathrm{L}^{-1} \mathrm{AlB}$} & \multicolumn{2}{|c|}{$3000 \mathrm{mg} \cdot \mathrm{L}^{-1} \mathrm{AIB}$} & \multirow[b]{2}{*}{ Média } \\
\hline & Anelamento & Decepa & Anelamento & Decepa & \\
\hline $\begin{array}{r}17 \\
\text { anos }\end{array}$ & 19,23 & 24,27 & 26,47 & 26,10 & $24,02 \mathrm{a}$ \\
\hline $\begin{array}{c}80 \\
\text { anos }\end{array}$ & $|3,5|$ & 15,23 & 18,12 & 15,30 & $15,54 \mathrm{~b}$ \\
\hline Média & \multicolumn{2}{|c|}{$18,06 \mathrm{~A}$} & \multicolumn{2}{|c|}{$21,50 \mathrm{~A}$} & \\
\hline
\end{tabular}

Coeficiente de variação $(\%)=22,12$

Médias seguidas da mesma letra minúscula na vertical e médias seguidas da mesma letra maiúscula na horizontal, não diferem significativamente entre si pelo teste de Tukey a $5 \%$ de probabilidade. 
A porcentagem de estacas com calos não foi influenciada pelos fatores estudados. O declínio nas porcentagens de enraizamento de espécies florestais está geralmente relacionado com os efeitos da maturação das plantas matrizes (FERREIRA et al., 20I0; WENDLING et al., 20l4b) onde, estacas provenientes de plantas mais jovens tendem a apresentar maior enraizamento em relação à plantas em estádio de maturação avançado e, consequentemente uma menor formação de calos (REINEKE et al., 2002).

A utilização de brotações provenientes de poda gera características morfológicas e fisiológicas juvenis fundamentais para a recuperação da competência celular e consequente enraizamento, tais como reduzida lignificação e elevada atividade cambial, resultantes da fase mais ativa de crescimento dos ramos (LIMA et al., 201।; WENDLING et al., 20/3), garantindo desse modo a manifestação do potencial genético do material selecionado.

Para a porcentagem de estacas vivas, apenas na concentração de $3000 \mathrm{mg} \cdot \mathrm{L}^{-1}$ AlB o anelamento foi inferior à decepa. $\mathrm{Na}$ concentração de $0 \mathrm{mg} \cdot \mathrm{L}^{-1} \mathrm{AlB}$, as matrizes de 17 anos foram inferiores às de 80 anos, enquanto que a concentração de $3000 \mathrm{mg} \cdot \mathrm{L}^{-1}$ AlB foi superior à testemunha nas matrizes de 17 anos (Tabela 4). De maneira geral, a porcentagem de estacas vivas foi baixa, em decorrência das

TABELA 4 Porcentagem de sobrevivência em estacas de erva-mate, provenientes de anelamento e decepa, em duas idades de plantas matrizes e dois tratamentos com regulador vegetal, realizados no inverno/2006, Colombo-PR.

TABLE 4 Survival percentage in cuttings of yerba mate from girdling and coppicing in two ages of mother plants and two plant growth regulator treatments, conducted in winter/2006, Colombo-PR.

\begin{tabular}{ccc}
\hline Método de & \multicolumn{2}{c}{ \% Sobrevivência } \\
\cline { 2 - 3 } $\begin{array}{c}\text { indução de } \\
\text { brotações }\end{array}$ & $0 \mathrm{mg} \cdot \mathrm{L}^{-1} \mathrm{AlB}$ & $3000 \mathrm{mg} \cdot \mathrm{L}^{-1} \mathrm{AlB}$ \\
\hline Anelamento & $2,50 \mathrm{a} \mathrm{A}$ & $0,00 \mathrm{~b} \mathrm{~A}$ \\
Decepa & $2,50 \mathrm{a} \mathrm{A}$ & $6,25 \mathrm{a} \mathrm{A}$ \\
Idade matrizes & $0 \mathrm{mg} \cdot \mathrm{L}^{-1} \mathrm{AlB}$ & $3000 \mathrm{mg} \cdot \mathrm{L}^{-1} \mathrm{AlB}$ \\
17 anos & $0,00 \mathrm{~b} \mathrm{~B}$ & $5,00 \mathrm{a} \mathrm{A}$ \\
80 anos & $5,00 \mathrm{a} \mathrm{A}$ & $1,25 \mathrm{a} \mathrm{A}$
\end{tabular}

Coeficiente de variação $(\%)=116,35$

Médias seguidas da mesma letra minúscula na vertical e médias seguidas da mesma letra maiúscula na horizontal, não diferem significativamente entre si pelo teste de Tukey a 5\% de probabilidade. altas taxas de enraizamento.

Os baixos valores obtidos para mortalidade podem ser considerados satisfatórios para a espécie. Isso sugere que as condições em que o experimento foi conduzido foram favoráveis para a manutenção da sobrevivência das estacas (STUEPP et al., 2013).

\section{Brotações provenientes de decepa realizada em árvores de mais de 80 anos coletadas no inverno/2006 e verão/2007}

A porcentagem de enraizamento foi influenciada pela época de instalação e interação entre época e regulador vegetal. $\mathrm{O}$ comprimento das três maiores raízes por estaca e porcentagem de estacas com calos tiveram influência apenas da época de instalação, enquanto a porcentagem de estacas mortas foi influenciada pelos dois fatores analisados, assim como pela interação entre eles.

$\mathrm{Na}$ concentração de $0 \mathrm{mg} \cdot \mathrm{L}^{-1} \mathrm{AlB}$, inverno e verão diferiram entre si, de modo que $\circ$ verão foi superior com $85 \%$ de estacas enraizadas (TABELA 5). Esta porcentagem de enraizamento pode ser considerada satisfatória para a erva-mate, uma vez que a média de enraizamento para esta espécie está abaixo deste valor (BITENCOURT et al., 2009; WENDLING et al., 2013).

O estabelecimento de cultivos clonais de ervamate a partir de plantas adultas é um desafio para os pesquisadores, limitado por uma série de fatores como a falta de métodos eficientes de rejuvenescimento e adequação das técnicas de manejo e propagação (WENDLING et al., 2007). Desta forma, o resultado de $85 \%$ de enraizamento obtido em estacas provenientes de plantas com mais de 80 anos de idade pode ser considerado um avanço na propagação de material adulto da erva-mate, não se descartando a possível influência positiva do material genético utilizado (MELO et al., 20I I), sobretudo por uma maior pré-disposição ao enraizamento das brotações revigoradas destas matrizes.

Os maiores valores para o número de raízes e sobrevivência foram de 1 I, 23 raízes, no verão com $0 \mathrm{mg} \cdot L^{-1}$ $\mathrm{AIB}$ e $5 \%$ de estacas vivas, em estaquia realizada no inverno com $0 \mathrm{mg} \cdot \mathrm{L}^{-1}$ AlB. O comprimento das três maiores ráizes foi maior no verão $(26,74 \mathrm{~mm}$ em média), diferindo significativamente do inverno (15,27 mm em média).

A porcentagem de estacas com calos foi maior no inverno $(38,75 \%)$, diferindo estatisticamente do verão (I I ,25\%) (Tabela 5). De maneira geral, o inverno apresentou menor enraizamento e maior indução de calos. Ainda no inverno, a mortalidade das estacas não diferiu entre as duas concentrações de AIB, porém no verão, foi superior na concentração de $3000 \mathrm{mg} \cdot \mathrm{L}^{-1} \mathrm{AlB}$ 
(I2,50\%). Para a concentração de $0 \mathrm{mg} \cdot \mathrm{L}^{-1}$ AlB não houve diferença entre as estações, enquanto na concentração de $3000 \mathrm{mg} \cdot \mathrm{L}^{-1}$ AIB, o verão diferiu significativamente do inverno, com maior porcentagem de mortalidade das estacas (Tabela 5). A mortalidade das estacas de erva-mate pode ser

TABELA 5 Porcentagem de enraizamento, comprimento médio das três maiores raízes, porcentagem de calos e mortalidade em estacas de erva-mate provenientes de decepa de árvores de mais de 80 anos, com aplicação de tratamentos com regulador vegetal (AIB), no inverno/2006 e verão/2007, Colombo-PR.

TABLE 5 Rooting percentage, length of the three longest roots per cutting, percentage of cuttings with callus and mortality in cuttings of yerba mate from coppicing of trees over 80 years old trees, applying treatments with plant growth regulators (AIB), conducted in winter/2006 and summer/2007, Colombo-PR.

\begin{tabular}{cccc}
\hline \multicolumn{4}{c}{$\%$ Enraizamento } \\
\hline & Inverno & Verão & Média \\
\hline $0 \mathrm{mg} \cdot \mathrm{L}^{-1} \mathrm{AIB}$ & 46,25 a B & 85,00 a A & 65,63 \\
\hline $3000 \mathrm{mg} \cdot \mathrm{L}^{-1}$ AIB & 67,50 a A & 73,75 a A & 70,63 \\
\hline Média & 56,88 & 79,38 \\
\hline
\end{tabular}

Coeficiente de variação $(\%)=22,40$

Comprimento médio das 3 maiores raízes por estaca $(\mathrm{mm})$

\begin{tabular}{lccc}
\hline & Inverno & Verão & Média \\
\hline $0 \mathrm{mg} \cdot \mathrm{L}^{-1}$ AIB & 5,23 & 25,60 & 20,42 \\
\hline $3000 \mathrm{mg} \cdot \mathrm{L}^{-1}$ AIB & 5,30 & 27,88 & 21,59 \\
\hline Média & $15,27 \mathrm{~B}$ & $26,74 \mathrm{~A}$ & \\
\hline
\end{tabular}

Coeficiente de variação $(\%)=22,12$

\begin{tabular}{lccc}
\hline \multicolumn{4}{c}{$\%$ Calos } \\
\hline & Inverno & Verão & Média \\
\hline $0 \mathrm{mg} \cdot \mathrm{L}^{-1} \mathrm{AlB}$ & 48,75 & $1 \mathrm{I}, 25$ & 30,00 \\
\hline $3000 \mathrm{mg} \cdot \mathrm{L}^{-1} \mathrm{AlB}$ & 28,75 & $1 \mathrm{I}, 25$ & 20,00 \\
\hline Média & $38,75 \mathrm{~A}$ & $\mathrm{I} \mathrm{I}, 25 \mathrm{~B}$ \\
\hline Coeficiente de variação $(\%)=44,37$ \\
\hline
\end{tabular}

\section{\% Mortalidade}

\begin{tabular}{lccc}
\hline & Inverno & Verão & Média \\
\hline $0 \mathrm{mg} \cdot \mathrm{L}^{-1}$ AIB & 0,00 a A & 2,50 b A & 1,25 \\
\hline $3000 \mathrm{mg} \cdot \mathrm{L}^{-1}$ AIB & $\mathrm{I}, 25$ a B & 12,50 a A & 6,88 \\
\hline Média & 0,63 & 7,50 & \\
\hline
\end{tabular}

Coeficiente de variação $(\%)=|3|, \mid 5$

Médias seguidas da mesma letra minúscula na vertical e médias seguidas da mesma letra maiúscula na horizontal, não diferem significativamente entre si pelo teste de Tukey a $5 \%$ de probabilidade. considerada baixa, chegando à nulidade na estaquia instalada no inverno, sem utilização do regulador vegetal.

\section{Brotações provenientes de anelamento e decepa coletadas no verão/2007}

Não foi verificado influência dos tratamentos em nenhuma das variáveis analisadas. A maior porcentagem de enraizamento, assim como o maior número de raízes por estaca foram observados em estacas coletadas de decepa, sem utilização de regulador vegetal, o que é importante no sentido de representar menor custo na produção das mudas. Além disso, o enraizamento satisfatório das brotações basais juvenis induzidas pelo anelamento e decepa pode ser atribuído à juvenilidade deste material.

$\mathrm{Na}$ transição da fase juvenil para adulta, as plantas passam por mudanças em seus meristemas que ocorrem em períodos distintos do desenvolvimento ontogenético (WENDLING et al., 20l4a). Contudo, algumas características relacionadas à juvenilidade são mantidas na região basal do caule de plantas maduras e, a capacidade de enraizamento é uma característica associada ao material juvenil (WENDLING et al., 20l4b), justificando deste modo as taxas de até $85 \%$ de enraizamento observadas em estacas provenientes de brotações revigoradas de matrizes com 80 anos de idade.

O maior comprimento de raízes foi observado nas estacas coletadas de decepa, com aplicação de $3000 \mathrm{mg} \cdot \mathrm{L}^{-1}$ AIB (28,08 mm). As estacas provenientes de anelamento, sem aplicação de AIB, apresentaram as maiores porcentagens de calos e sobrevivência, o que se deve à menor porcentagem de enraizamento observada neste tratamento e também é uma característica marcante da maturidade celular. $\mathrm{O}$ tratamento de decepa, com $3000 \mathrm{mg} \cdot \mathrm{L}^{-1} \mathrm{AlB}$, foi o que apresentou a maior mortalidade das estacas (I5\%).

A carência em métodos eficientes de rejuvenescimento de material adulto é um fator limitante à estaquia em escala comercial de erva-mate (WENDLING et al., 2007). Com base nestes resultados, pode-se afirmar que as técnicas de anelamento e decepa foram eficientes no revigoramento dos brotos emitidos, resultando em ótimos resultados para enraizamento, mesmo utilizando matrizes com mais de 80 anos de idade.

\section{CONCLUSÕES}

método de indução das brotações por meio de anelamento e decepa de erva-mate foi eficiente para o revigoramento e sucesso do enraizamento; recomendando-se o uso de AIB somente em estacas provenientes de matrizes mais velhas (superior a 80 anos). 


\section{REFERÊNCIAS}

ALMEIDA, F.D.de; XAVIER, A.; DIAS, J.M.M. Propagação vegetativa de árvores selecionadas de Eucalyptus cloeziana F. Muell. por estaquia. Revista Árvore, Viçosa, v.3I, n.3, p.445-453, 2007.

BITENCOURT, J.; ZUFFELLATO-RAIBS, K.C.; KOEHLER, H.S. Estaquia de Ginkgo biloba L. utilizando três substratos. Revista Brasileira de Plantas Medicinais, Botucatu, v. 12, n.2, p.135-140, 2010.

BITENCOURT, J.; ZUFFELLATO-RAIBS, K.C.; WENDLING, I.; KOEHLER, H.S. Enraizamento de estacas de erva-mate (llex paraguariensis St. Hill) provenientes de brotações rejuvenescidas. Revista Brasileira de Plantas Medicinais, Botucatu, v. I I, p.277-28I, 2009.

BRONDANI, G.E.; ARAUJO, M.A.; WENDLING, I.; KRATZ, D. Enraizamento de miniestacas de erva-mate sob diferentes ambientes. Pesquisa Florestal Brasileira, Colombo, n.57, p.29-38, 2008.

DARTORA, N.; SOUZA, L.M.; PAIVA, S.M.; SCOPARO, C.T.; IACOMINIA, M.; GORINA, P.A.J.; RATTMANN, T.D.; SASSAKI, G.L. Rhamnogalacturonan from llex paraguariensis: A potential adjuvant in sepsis treatment. Carbohydrate Polymers, Tenbury Wells, v.92, n.2, p. I776-I 782, 2013.

FERREIRA, B.G.A.; ZUFFELLATO-RAIBS, K.C.; WENDLING, I.; KOEHLER, H.S.; NOGUEIRA, A.C. Miniestaquia de Sapium glandulatum (vell.) pax com o uso de ácido indol butírico e ácido naftaleno acético. Ciência Florestal, Santa Maria, v.20, n.I, p.19-31, 2010.

HUSEN, A. Changes of soluble sugars and enzymatic activities during adventitious rooting in cuttings of Grewia optiva as affected by age of donor plants and auxin treatments. Am J Plant Physiol, New York, v.7, p. I-16, 2012.

LIMA, D.M.; BIASI, L.A.; ZANETTE, F.; ZUFFELLATORIBAS, K.C.; BONA, C.; MAYER, J.L.S. Capacidade de enraizamento de estacas de Maytenus muelleri Schwacke com a aplicação de ácido indol butírico relacionada aos aspectos anatômicos. Revista Brasileira de Plantas Medicinais, Botucatu, v. I3, n.4, p.422-438, $201 \mathrm{l}$.

MELO, L.A.; DAVIDE, A.C.; TEIXEIRA, L.A.F. Methodology for stock plants rescue and cuttings rooting of Eremanthus erythropappus. Revista Cerne, Lavras, v. I8, n.4, 2012.

MELO, L.A.; XAVIER, A.; PAIVA, H.N.; BORGES, S.R. Otimização do tempo necessário para o enraizamento de miniestacas de clones híbridos de Eucalyptus grandis. Rev. Árvore, Viçosa, v.35, n.4, p.759-767, 201 I.
PAGLIOSA, C.M.; VIEIRA, M.A.; PODESTÁ, R.; MARASCHIN, M.; ZENI, A.L.B.; AMANTE, E.R.; AMBONI, R.D.M.C. Methylxanthines, phenolic composition, and antioxidant activity of bark from residues from mate tree harvesting (Ilex paraguariensis A. St. Hil.). Food Chemistry, Reading, v. I22, n.I, p.I73-178, 2010

PIJUT, P.M.; WOESTE, K.E.; MICHLER, C.H. Promotion of adventitious root formation of difficult-to-root hardwood tree species. Horticultural Reviews, West Lafayette, v.38, p.2I3-25I, 2011 .

POP, T.I.; PAMFIL, D.; BELLINI, C. Auxin Control in the Formation of Adventitious Roots. Notulae Botanicae Horti Agrobotanici, Cluj-Napoca, v.39, n.I, p.307-316, 2011.

REINEKE, R.A.; HACKETT, W.P.; SMITH, A.G. Lignification associated with decreased adventitious rooting competence of English ivy petioles. Journal of Environmental Horticulture, Washington, v.20, p.236-239, 2002.

STUEPP, C.A.; PEREIRA, G.P.; ZEM, L.M.; PEÑA, M.L.; BUENO, P.M. C.; SPADER, V.; ZUFFELLATO-RAIBS, K.C.; ROSA, G.M. Enraizamento de melaleuca: influência da altura de coleta das estacas e aplicação de AIB. Colloquium Agrariae, Presidente Prudente, v.9, p.0I-09, 2013.

STUEPP, C.A.; ZUFFELLATO-RIBAS, K.C.; WENDLING, I.; KOEHLER, H.S.; BONA, C. Vegetative propagation of mature dragon trees through epicormic shoots. Revista Bosque, Valdivia, v.35, n.3, p.333-34I, 2014

TITON, M.; XAVIER, A.; OTONI, W.C. Clonal propagation of Eucalyptus grandis using the mini-cutting and microcutting techniques. Scientia Florestalis, Piracicaba, n.7I, p. 109-17, 2006.

WENDLING, I.; DUTRA, L.F.; GROSSI, F. Produção e sobrevivência de miniestacas e minicepas de ervamate cultivadas em sistema semi-hidropônico. Pesquisa Agropecuária Brasileira, Brasília, v.42, p.289-292, 2007.

WENDLING, I.; BRONDANI, G.E.; BIASSIO, A.; DUTRA, L.F. Vegetative propagation of adult llex paraguariensis trees through epicormic shoots. Acta Scientiarum, Maringa, v.35, n.I, p.II7-125, 2013

WENDLING, I.; TRUEMAN, S.J.; XAVIER, A. Maturation and related aspects in clonal forestry-Part I: concepts, regulation and consequences of phase change. New Forests, San Francisco, v.I, p.I-23, 20I4a.

WENDLING, I.; TRUEMAN, S; XAVIER, A. Maturation and related aspects in clonal forestry-part II: reinvigoration, rejuvenation and juvenility maintenance. New Forests, San Francisco, v. I, p.I-I4, 2014b. 
